\title{
The new challenges in ST-Segment Elevation Acute Myocardial Infarction in Mexico: "The IMSS Infarction Code"
}

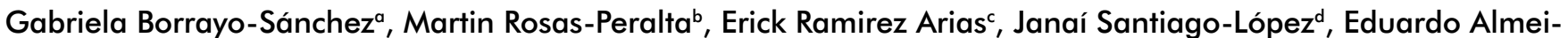 \\ da-Gutiérrez ${ }^{e}$, Hector David Martínez-Chapa ${ }^{f}$ Jose de Jesús Arriaga-Dávila ${ }^{g}$ \\ aHead of Evaluation and Accountability Division for Medical Care, CUMAE-IMSS, Mexico \\ bHead of Special Projects Area, Coordination of High Specialty Units; CUMAE-IMSS, Mexico \\ cHead of Emergency Room. Hospital of Cardiology of the National Medical Center, XXI Century, IMSS Mexico \\ dSpecial Projects Area. CUMAE-IMSS, Mexico \\ eTitular of the Directorate of Education and Research in Health of the Hospital of Cardiology of the National Medical Center XXI century, IMSS Mexico \\ Titular of Medical Attention Unit, IMSS Mexico \\ ghead of Medical Benefits of IMSS, Mexico
}

Article Info

\section{Article Notes}

Received: October 09, 2017

Accepted: November 03, 2017

\section{*Correspondence:}

DC Martin Rosas Peralta, Head of Special Projects Area, Coordination of High Specialty Units, IMSS. Durango 289-1A Colonia Roma. Cuauhtémoc Delegation, 06700 México, Tel: 57261700 ext. \# 16965, México

E-mail:mrosas_peralta@hotmail.com

(c) 2017 Rosas-Peralta M. This article is distributed under the terms of the Creative Commons Attribution 4.0 International License.

\section{Keywords}

Infarction Code

Acute Myocardial Infarction

\section{ABSTRACT}

Mexican Institute of Social Security is the principal institution of health in México, actually 75 million of Mexican people is covered. Infarction Code strategy has been recently applied at the national level. We inform the news of international guides for ST-segment elevation acute myocardial infarction and how we make the corresponding adaptions into the Infarction Code in Mexico. The implementation of this strategy in Mexico has resulted in a decrease in mortality by more than $50 \%$. (From $26 \%$ to $8 \%$; one year after its implementation)

\section{Introduction}

In Mexico as the rest of the world the main cause of death is cardiovascular disease where ischemic heart disease plays a central role $^{1-5}$. The adequacy and adaptations of international guidelines for the care of patients suffering from ST segment elevation acute myocardial infarction (STEMI), reminds to be a continuous challenge ${ }^{1}$. Thus, in the medical attention not all are scientific concepts, the processes for its clinical implementation needs to be considered. Any recommendations or international guide should serve as reference framework to generate specific strategies in each country. Above all countries like Mexico where there is sub optimal number of Angioplasty Units. To make a good map of processes that guarantee the adequate care of STEMI in accordance with international guidelines and just in time, it will be necessary to contextualize the clinical, hemodynamic and infrastructural health context including all health team from the first medical contact to specific therapy. In this sense most recent European guideline for STEMI care headed by far the operational definition of the processes that are involved in the care and the prognosis of this entity. The aims of this work were to review the news on STEMI guideline from European Society of Cardiology, show the strategy named "infarction code" in Mexico and the impact to implement in a correct manner the international guidelines. 


\section{Recent changes on STEMI guideline by the} European Society of Cardiology (ESC) ${ }^{1}$

For the first time in ESC guideline for STEMI there is a clear definition of when to start the clock from the goal of 90 minutes to treat patients with percutaneous coronary intervention (PCI). The clock should begin at the moment of the diagnosis of STEMI by electrocardiogram (ECG) ("zero time"). There was confusion about whether the clock started when the patient has symptoms, when it calls for emergency services, when the ambulance arrives at the scene, or when the patient arrived at the hospital. I am so clear that all the processes of health care should be initiated to the ECG. However insists on that the clinical suspicion is the keystone.

The vague term of door to balloon has been removed from the guidelines and the first medical contact (FMC) is defined as the moment when the patient is initially evaluated by a physician, paramedic or nurse that gathers and interprets the ECG.

In cases where fibrinolysis is the strategy of reperfusion, the maximum delay time from diagnosis of STEMI treatment has reduced from 30 minutes to 10 minutes by 2017.

Complete revascularization is not recommended in 2012 document that says that they should be treated only the infarct-related arteries. The current guidelines indicate that it should be considered complete revascularization, even in non-infarct-related arteries and treat them during the procedure of ICP or other time prior to discharge.

Thrombus aspiration is no longer recommended, based on two large trials in more than 15,000 patients. Also not recommended differ Stenting, involving open the artery and wait 48 hours to implant a stent. With respect to PCI, the use of Drug Eluting Stents instead of only metal has gained a stronger recommendation that has the use of radial approach, instead of femoral arterial access.

When it comes to drugs, the dual antiplatelet therapy beyond 12 months in selected patients can be considered. Bivalirudin was downgraded from class I and IIa, enoxaparin class IIa to IIb rating raised. Cangrelor, which was not mentioned in the document of 2012, has been recommended as an option in certain patients. Another novelty is a recommendation for additional therapy in patients with high cholesterol despite taking maximum doses of Statins lowering.

The critical point for the start of the administration of oxygen therapy less than $95 \%$ has been reduced to less than $90 \%$. Left and right bundle branch block are considered equal to recommend emergency angiography when patients have ischemic symptoms. A chapter of myocardial infarction with coronary arteries without obstructive lesions (MINOCA) was added, comprising up to $14 \%$ of patients with STEMI and requires additional diagnostic tests and tailored therapy that may differ from typical STEMI. Another new concept is the inclusion of quality indicators, for the evaluation of the process of care in the health system.

\section{Infarction Code, IMSS-México ${ }^{6-9}$}

The strategy "IMSS infarction code" emerges as an urgent need to combat the very high reported mortality in Mexico secondary to STEMI.

In February 2015 began the strategy code infarction in the Mexican Institute of Social Security (IMSS), even though the concept of permeable artery as soon as possible is not a new concept, the standardization of processes in the prompt medical attention to reduce time and select the best reperfusion strategy either thrombolysis for PCI after or primary PCI is what characterizes this strategy.

Today it boasts 13 centers of reperfusion with hemodynamics room for ICP and the goal is to cover the 23 centers nationwide. However she has trained to 194 units of health care in three levels of 25 IMSS delegations.

The greatest impact of the strategy was quickly in the reduction of mortality as a basal rate of $26 \%$ (OCDE) was down $10 \%$ (8 to 12\%, 2017-IMSS). Figure 1 shows the evolution in time of the different strategies, being the most notorious finding the remarkable increase in primary angioplasty ${ }^{10-13}$.

\section{Regulatory Centre}

Another important development was the integration of regulatory centers that automated consultation and management services. Using telemedicine the cases are selected and transferred in a more efficient manner. (Figure 2)

Our current record until July 2017 that includes 2 years form implementation of the "infarction code" strategy at IMSS is 7,881 cases with an overall mortality of $10 \%$ (8 to $12 \%)$. Average response time for diagnosis was reduced $50 \%$ and profit increased remarkably in the number of cases to be transferred to hospital for primary PCI.

\section{No Return Point into the Infraction Code in IMSS- Mexico}

Because of the very encouraging results since the beginning of the strategy, code infarction IMSS authorities granted facilities for implementation across the country. So after a situational diagnosis in all aspects, clinical, methodological, processes management, engineering and administration set a goal for 2018. The strategy was entitled "No Return Point".

We know that in the STEMI, reperfusion therapy has become a predictor of survival in the short and long 


\subsection{Thirty-day mortality after admission to hospital for AMI based on admission data, 2003 to 2013 (or nearest years)}

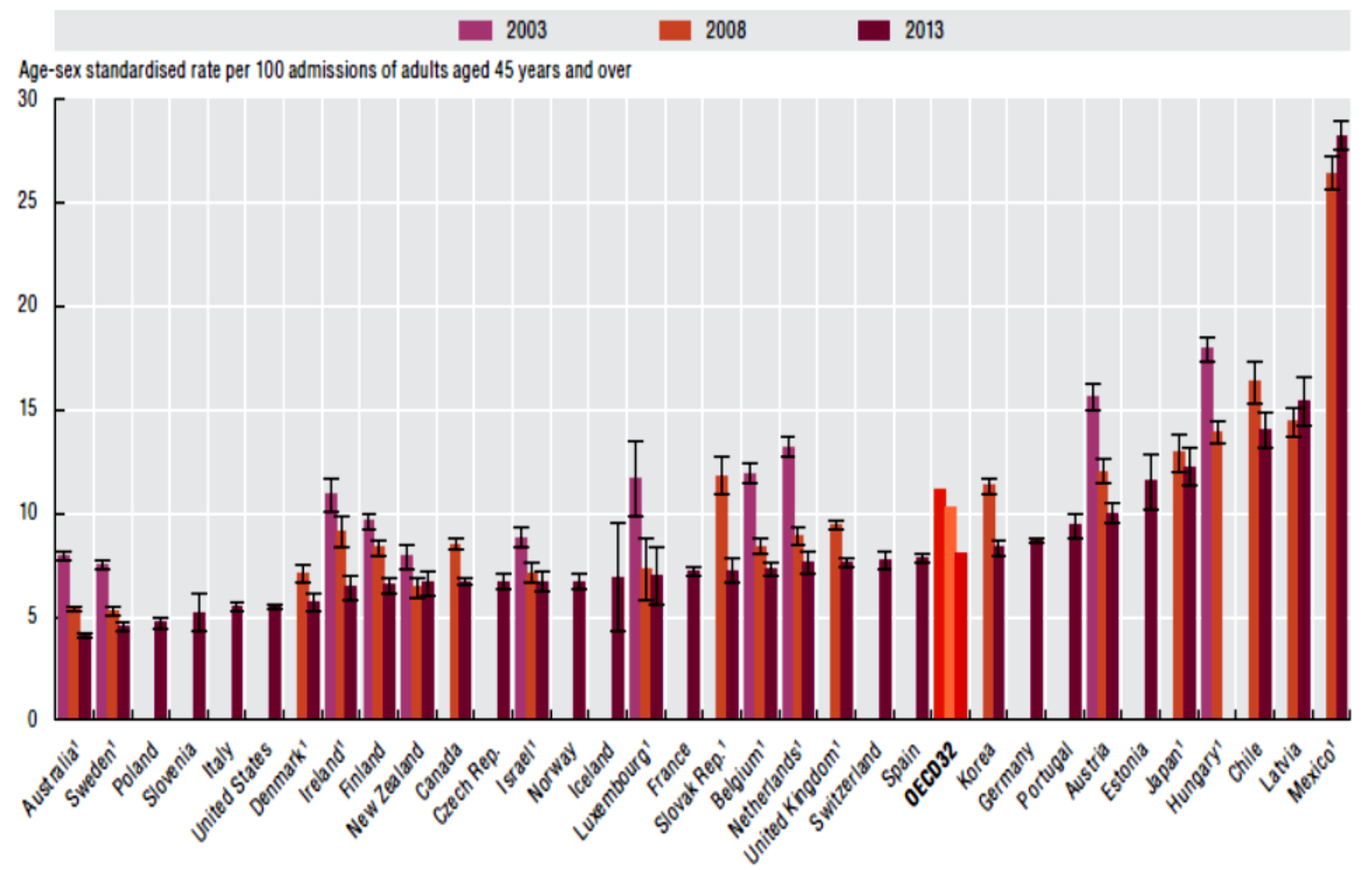

Note: $95 \%$ confidence intervals represented by $\mathrm{H}$. Three-year average for Iceland and Luxembourg.

1. Admissions resulting in a transfer are included.

Source: OECD Health Statistics 2015, http///dx.doi.org/10.1787/health-data-en.

StatLink http://dx.doi.org/10.1787/888933281135

Figure 1: Mortality rate due to Acute Myocardial Infarction in Mexico compared with other countries.

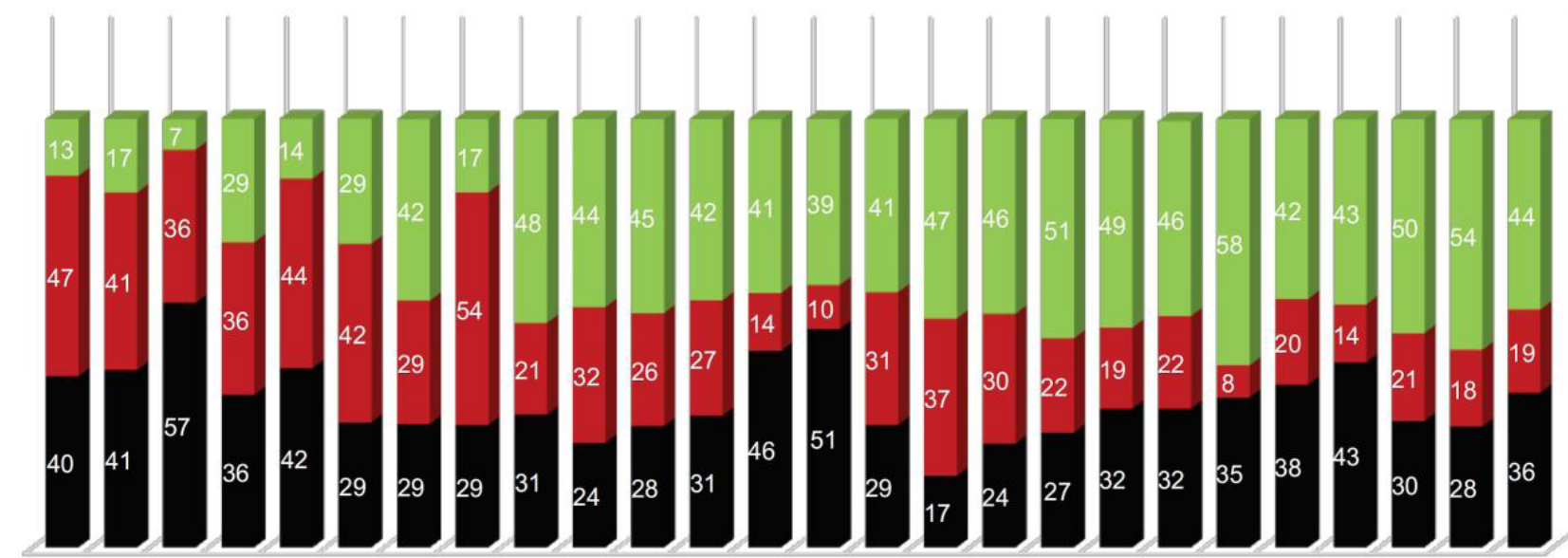

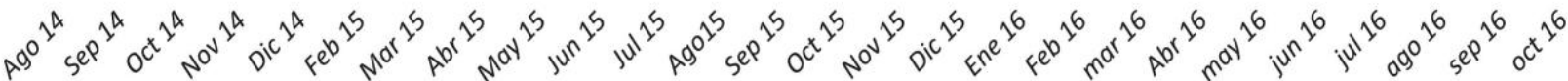

No Reperfusion strategy $\square$ Fribrinolytic Strategy Primary PCI Strategy

Figure 2. Evolution on time of different strategies of reperfusión in Mexico, before and after Infarct Code implementation (Feb, 2015) (IMSS). 
term ${ }^{11-14}$. Attachment to the clinical practice guidelines care regionalized through the implementation of the code demonstrated that reperfusion therapyreduces significantly hospitalization days, reduce complications and risk of infarction recurrence, other mechanical complications and death. It was also noted that the standardization of care protocol reduce the percentage of patients who remained outside of reperfusion treatment, increased PCI, the rate which has impact on the reduction of complications. These findings are similar to the initial studies of Fibrinolytic therapy (GISSI ${ }^{15}$, ISIS- $2^{16}$, Maastricht ${ }^{17}$ ), in terms of a significant reduction in mortality and complications. The strategy code infarction is the first its kind in our country. We are working to implement twelve network more into the country, in order to standardize it and reach total coverage. A timely follow-up of each case will be made more opportunity areas will be developed to improve the process $^{18}$.

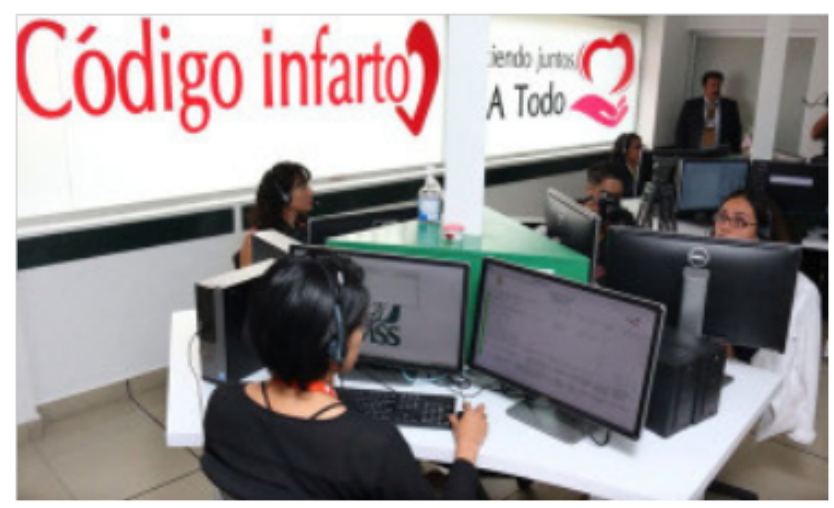

Figure 3. Infarction Code regulatory center in Mexico.

\section{No Return Point- Infarction Code}
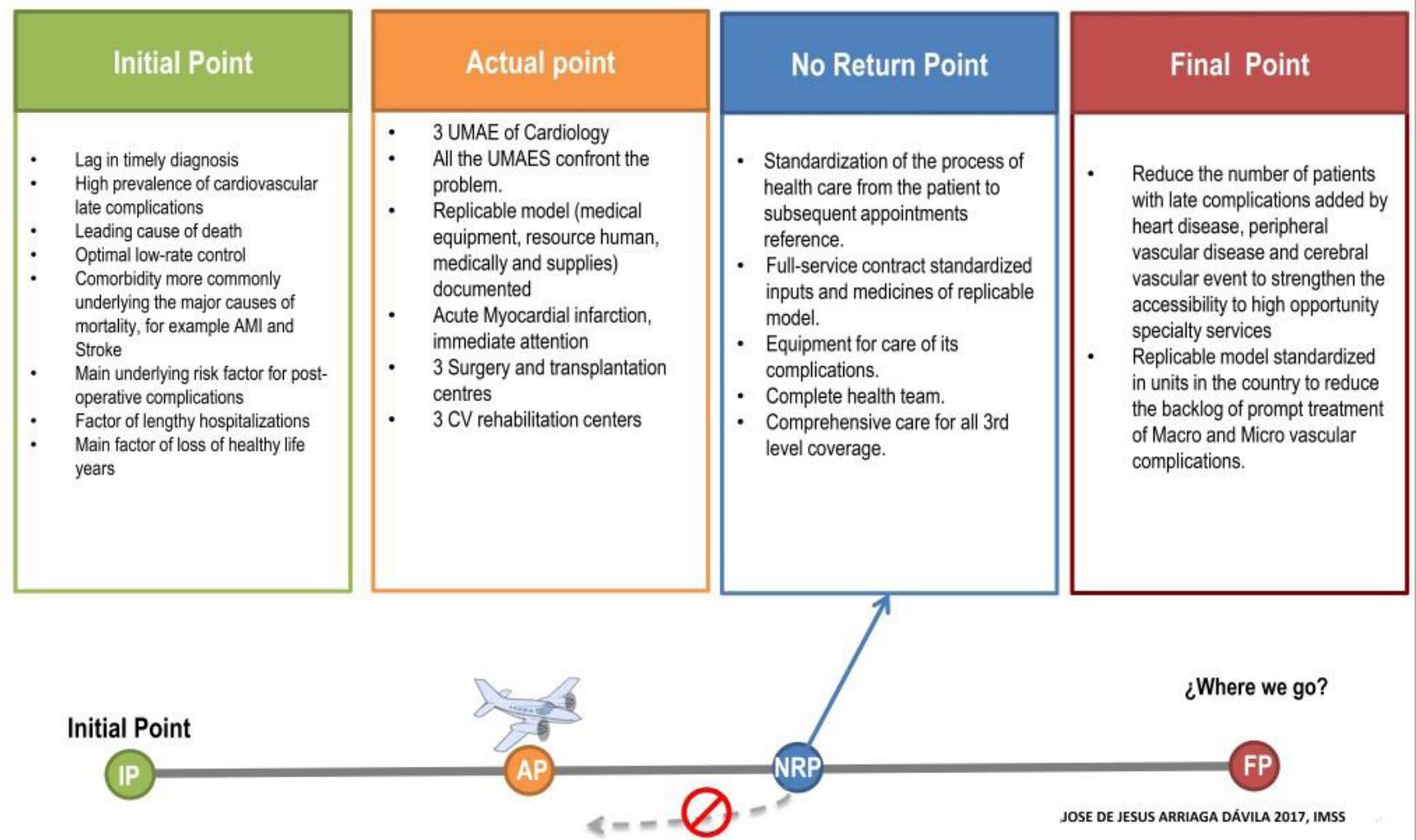

Figure 4. No Return Strategy for Infarction Code. IMSS 2017. 


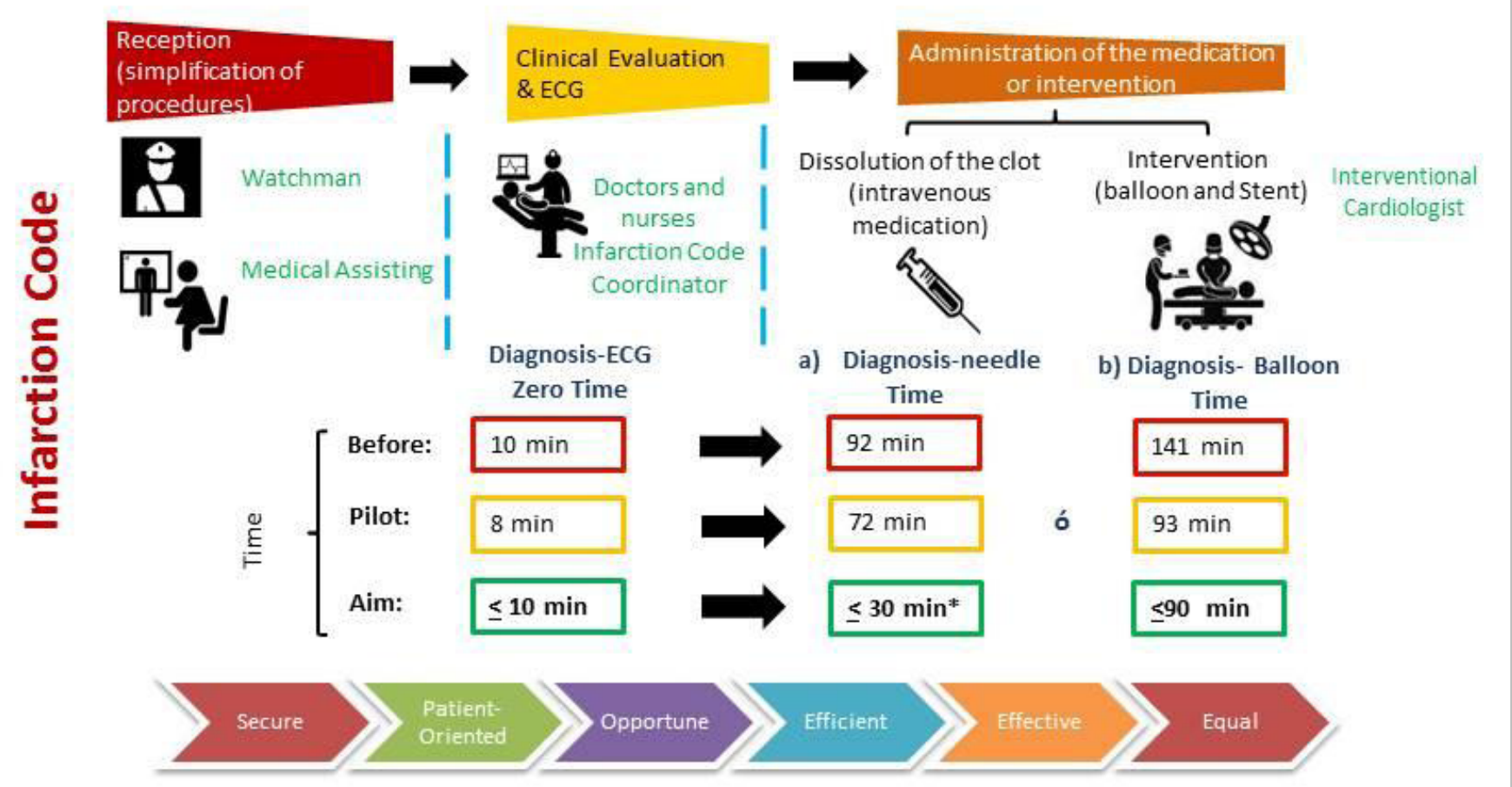

* Ideally $<10$ minutes

Figure 5. Infarction Code: Quality Focus based on six dimensions in Mexico (Borrayo SG, IMSS-2017).

\section{Conclusions}

The implementation of the infarction code in IMSSMexico allowed an improvement of the reperfusion strategies for STEMI, and the most important aspect, time was significantly reduced ( $>50 \%)$. Fewer early complications and less death have been observed. The international guidelines should be adapted to the local circumstances in a continuous manner in each country of the world, Mexico have a great challenge.

\section{References}

1. The Task Force for the management of acute myocardial infarction in patients presenting with ST-segment elevation of the European Society of Cardiology (ESC). 2017 ESC Guidelines for the management of acute myocardial infarction in patients presenting with ST-segment elevation. European Heart Journal (2017) 00,1-66. https://academic. oup.com/eurheartj/article-abstract/doi/10.1093/eurheartj/ ehx393/40950 $42 / 2017$

2. Organización para la AQ3 Cooperación y el Desarrollo Económicos (OCDE). 2015.

3. Instituto Nacional de Estadística, Geografía e Informática. Epidemiología de la defunción poblacional. México: INEGI; 2015. Disponible en: www. inegi.gob.mx

4. Keeley EC, Boura JA, Grines CL. Primary angioplasty versus intravenous thrombolytic therapy for acute myocardial infarction: a quantitative review of 23 randomized trials. Lancet. 2003; 361: 13-20.

5. Steg PG, Bonnefoy E, Chabaud S, et al. Comparison of Angioplasty and Prehospital Thrombolysis In acute Myocardial infarction (CAPTIM) Investigators. Impact of time to treatment on mortality after prehospital fibrinolysis or primary angioplasty: data from the CAPTIM randomized clinical trial. Circulation. 2003; 108: 2851-6.

6. Pinto DS, Kirtane AJ, Nallamothu BK, et al. Hospital delays in reperfusion for ST elevation myocardial infarction: implications when selecting a reperfusion strategy. Circulation. 2006; 114: 2019-25.

7. The task force on the management of ST-segment elevation acute myocardial infarction of European Society of Cardiology (ESC). ESC Guidelines for the management of acute myocardial infarction in patients presenting with ST-segment elevation. Eur Heart J. 2012; 33: 2569-619.

8. 2014 AHA/ACC Guideline for the management of patients with nonST-elevation acute coronary syndromes. A report of the American College of Cardiology/American Heart Association Task Force on Practice Guidelines. Circulation. 2014; 130: e344-e426.

9. Borrayo-Sánchez G, Madrid-Miller A, Arriaga-Nava R, et al. Riesgo estratificado de los síndromes coronarios agudos. Resultados del primer RENASCA-IMSS. Rev Med Inst Mex Seguro Soc. 2010; 48: 259-64.

10. Xavier D, Pais P, Devereaux PJ, et al. for the TN-STEMI Programme Investigators. CREATE registry investigators. Treatment and outcomes of acute coronary síndromes in India (CREATE): a prospective analysis of registry data. Lancet. 2008; 371: 1435-42.

11. Borrayo SG, Martínez MOG, Gudiño TA, et al. Código Infarto. Protocolo para servicios de urgencias. México Primera edición ISBN Obra independiente. 2015; 978-607-8392-42-1.

12. Krumholz HM, Wang Y, Mattera JA, et al. An administrative claims model suitable for profiling hospital performance based on 30-day mortality rates among patients with an acute myocardial infarction. Circulation. 2006; 113: 1683-92.

13. Seghieri C, Mimmi S, Lenzi J, et al. 30-day in-hospital mortality after acute myocardial infarction in Tuscany (Italy): an observational study using hospital discharge data. BMC Med Res Methodol. 2012; 12: 170 
14. Ross JS, Maynard C, Krumholz HM, et al. Use of administrative claims models to assess 30-day mortality among Veterans Health Administration hospitals. Med Care. 2010; 48: 652-8.

15. Hayashida K, Imanaka Y, Sekimoto M, et al. Evaluation of acute myocardial infarction in-hospital mortality using a risk-adjustment model based on Japanese administrative data. J Int Med Res. 2007; 35: 590-6.

16. Gruppo Italiano per lo Studio Della Streptochinasi nell'Infarcto Miocardio (GISSI). Effectiveness of intravenous thrombolytic treatment in acute myocardial infarction. Lancet. 1986; 1: 397-402.
17. Vermeer F, Oude Ophuis AJM, van den Berg, et al. Prospective randomized comparison between thrombolysis, rescue PCTA, and primary PTCA in patients with extensive myocardial infarction admitted to a hospital without PTCA facilities: a safety and feasibility study. Heart. 1999; 82: 426-31.

18. Larson DM, Duval S, Sharkey SW, et al. Safety and efficacy of a pharmaco-invasive reperfusion strategy in rural ST-elevation myocardial infarction patients with expected delays due to long-distance transfers. Eur Heart J. 2012; 33: 1232-40. 